\title{
CRIANÇAS QUE VIVEM COM AIDS E SUAS EXPERIÊNCIAS COM O USO DE ANTIRRETROVIRAIS
}

\author{
Joel KUYAVA , Eva Neri Rubim PEDRO ${ }^{b}$, Daisy Zanchi de Abreu BOTENE
}

\section{RESUMO}

Estudo com o objetivo de conhecer como a criança que vive com AIDS descreve sua experiência com o uso de antirretrovirais (ARV). Trata-se de um estudo qualitativo, exploratório, descritivo, realizado no ambulatório do Serviço de Assistência Especializada em Doença Sexualmente Transmissível, em Porto Alegre, no período entre maio e julho de 2009, com crianças em uso de ARV. A coleta das informações foi realizada utilizando-se a técnica de grupo focal. A análise, feita por meio da análise de conteúdo, evidenciou as seguintes categorias: modo de vida da criança com AIDS; manuseio da medicação; expectativas e sentimentos. O estudo trouxe contribuições importantes que ainda devem ser aprofundadas em relação a essa temática no universo infantil. A adesão aos ARV constitui-se um desafio para os profissionais da saúde e para a família. O conhecimento das vivências e estratégias das crianças pode oportunizar novas formas de ver, tratar e cuidar dessa parcela da população.

Descritores: Criança. Síndrome de Imunodeficiência Adquirida. HIV. Enfermagem.

\section{RESUMEN}

Estudio con el objetivo de conocer cómo el niño que vive con SIDA describe su experiencia con el uso de antirretrovirales (ARV). Es un estudio cualitativo, exploratorio y descriptivo realizado en el ambulatorio del Servicio de Asistencia Especializada en Enfermedades Sexualmente Transmisibles, en Porto Alegre, entre mayo y julio de 2009, con niños en tratamiento con ARV. La recolección de informaciones fue realizada utilizándose la técnica de grupo focal. El análisis, hecho por medio del análisis de contenido, evidenció las siguientes categorías: Modo de vida del niño con SIDA; Manejo de la medicación; Expectativas y sentimientos. El estudio trajo contribuciones importantes que todavía se deben profundizar en cuanto a esta temática en el universo infantil. La adhesión a los ARV constituye un desafio para los profesionales de la saludy para la familia. El conocimiento de las vivencias y estrategias de los niños puede suscitar nuevas formas de ver, tratar y cuidar a este segmento de la población.

Descriptores: Niño. Síndrome de Inmunodeficiencia Adquirida. VIH. Enfermería.

Título: Niños que viven con SIDA y sus experiencias con el uso de antirretrovirales.

\section{ABSTRACT}

The purpose of this study was to find out how children who live with AIDS describe their experiences with antiretrovirals (ARV). This qualitative exploratory-descriptive study was conducted in the outpatient clinic of the STD Specialized Care Service, in the city of Porto Alegre, between May and July 2009, with children using ARV. Information was collected adopting the focus group method. The analysis was carried out by means of content analysis, which has evidenced the following categories: Way of life of children with AIDS; Medication handling; Expectations and feelings. The study made important contributions that must be further analyzed in relation to this theme in the child's universe. The adherence to ARV constitutes a challenge for both health professionals and the family. Knowing more about children's experiences and strategies can provide new ways of seeing, treating, and caring for this segment of the population.

Descriptors: Child. Acquired Immunodeficiency Syndrome. HIV. Nursing.

Title: Children living with AIDS and their experiences with antiretroviral therapy.

\footnotetext{
a Enfermeiro. Mestrando em Enfermagem pelo Programa de Pós Graduação em Enfermagem da Escola de Enfermagem da Universidade Federal do Rio Grande do Sul. Porto Alegre, Rio Grande do Sul, Brasil.

b Enfermeira. Doutora em Educação. Professora Associada da Escola de Enfermagem da Universidade Federal do Rio Grande do Sul. Porto Alegre, Rio Grande do Sul, Brasil.

c Enfermeira. Doutoranda em Enfermagem pelo Programa de Pós Graduação em Enfermagem da Escola de Enfermagem da Universidade Federal do Rio Grande do Sul. Porto Alegre, Rio Grande do Sul, Brasil.
} 


\section{INTRODUÇÃO}

A epidemia da Síndrome da Imunodeficiência Adquiria (AIDS) no Brasil, atualmente representa um contingente 608 mil casos notificados de pessoas contaminadas pelo Vírus da Imunodeficiência Humana (HIV), desde 1980 até 2011. Do número total de casos confirmados, cerca de 210 mil $(34,6 \%)$ são mulheres. Cerca de $90 \%$ dos casos de crianças infectadas pelo HIV no Brasil, se deve à categoria de exposição vertical. No período entre 2000 e 2011 foram notificadas 61,789 gestantes soropositivas para o HIV, sendo a Região Sul a segunda região com maior número de gestantes soropositivas, ficando atrás, apenas, da Região Sudeste. E, no ano de 2011, 90,4\% dos casos de aids em crianças menores de 13 anos foram devido à transmissão vertical ${ }^{(1)}$.

Sabe-se que o atendimento de crianças nascidas de mães com HIV deve ser realizado em unidades especializadas, pelo menos até a definição de seu diagnóstico. As crianças que se revelarem infectadas deverão permanecer em atendimento nessas unidades. Em países desenvolvidos, têm sido recomendado visitas periódicas, mesmo às crianças que soroconverteram, até o final da adolescência, em virtude de terem sido expostas ao HIV e aos ARVs durante o período intra-uterino. Essa preocupação é em função do desconhecimento das possíveis repercussões da exposição a tais medicamentos a médio e longo $\operatorname{prazo}^{(2)}$.

Os avanços na terapia ARV permitiram uma melhora significativa na sobrevida e qualidade de vida das crianças e adolescentes com diagnóstico de infecção pelo HIV. Contudo, na prática clínica, um dos maiores obstáculos ao sucesso da terapia ARV é a má adesão dos pacientes e/ou familiares ao tratamento prescrito $^{(3)}$. Para que um paciente apresente uma adesão adequada é necessário que ele utilize os medicamentos prescritos, em pelo menos $80 \%$ de seu total, com a observância dos horários, doses e o tempo de tratamento recomendado ${ }^{(4)}$.

A terapêutica ARV deve ser usada criteriosamente, do contrário, pode-se incorrer no risco da indução de resistência e, conseqüentemente, no esgotamento precoce do arsenal ARV disponível ${ }^{(3)}$. Nas crianças e adolescentes com infecção pelo HIV a má-adesão a terapia é semelhante às dificuldades observadas nos adultos, tais como complexidade dos esquemas, várias tomadas diárias, interferência da alimentação, necessidade de conservação em geladeira, preparo trabalhoso e manifestações de intolerância; ao lado de outras dificuldades características da faixa etária, por exemplo a palatabilidade dos remédios ${ }^{(2)}$.

Diante desses fatos, antes do início da terapia ARV deve ficar claro para a família que o cumprimento rigoroso da prescrição médica é um fator fundamental para a eficácia do tratamento e que o uso irregular ou abandono prejudica o tratamento atual e compromete opções futuras. Na introdução da terapia é importante esclarecer dúvidas e temores com relação aos efeitos adversos, investigar a rotina da vida familiar e da criança e discutir sobre as possibilidades terapêuticas ${ }^{(2)}$.

No serviço de assistência especializada em Doenças Sexualmente Transmissíveis, DST/AIDS, ambulatório do Serviço de Assistência Especializada em DST/AIDS no Centro de Saúde Vila dos Comerciários, em Porto Alegre/RS, observa-se muitas vezes, queixas dos cuidadores em relação às dificuldades em administrar a medicação, o que em alguns casos, pode haver o risco de suprimi-la em virtude dessa situação. Essas dificuldades incluem o não querer ingerir, regurgitar, a fuga nos horários das medicações, entre outras. Sabe-se que é uma preocupação dos serviços e dos profissionais da saúde a necessidade de uma boa adesão para o sucesso do tratamento e sobrevida dessas crianças. Tal situação despertou nos pesquisadores o interesse em conhecer melhor essas crianças, seus cuidadores e contextos de vida.

Nesse sentido o estudo teve como objetivo conhecer como a criança que vive com aids descreve a sua experiência com o uso de antirretrovirais.

\section{METODOLOGIA}

Propôs-se encontros com um grupo de crianças, a fim de realizar-se um estudo, nos quais as suas falas por meio das brincadeiras e jogos, pudessem oportunizar o alcance de algumas dessas respostas e talvez indicar novos caminhos para o cuidado profissional e familiar. O estudo seguiu um delineamento exploratório descritivo, cuja finalidade foi o de observar, descrever e comprovar aspectos de uma situação $^{(5)}$. A metodologia utilizada foi qualitativa, partindo do fundamento que a realidade vai além dos fenômenos percebidos pelos nossos sentidos, os dados qualitativos trazem para o interior da análise, o subjetivo e o objetivo, os atores sociais e 
o próprio sistema de valores do cientista, os fatos e seus significados, a ordem e os $\operatorname{conflitos}^{(6)}$.

O estudo foi realizado no serviço de assistência especializada em DST/aids no Centro de Saúde Vila dos Comerciários, Porto Alegre/RS. A escolha por esse local de pesquisa se deu pelo fato do mesmo ser reconhecido como referência no atendimento em DST/aids.

Os participantes do estudo foram crianças entre nove e 11 anos, que atenderam os seguintes critérios inclusão: crianças que tivessem conhecimento do seu diagnóstico, disponibilidade de irem nos encontros, estivessem em uso de ARV e em acompanhamento no ambulatório de adesão.

O número inicial de participantes selecionados foi de nove crianças, porém o estudo foi realizado com quatro dessas crianças.

A coleta das informações foi realizada por meio da técnica do grupo focal com atividades lúdicas no período entre maio a julho de 2009. Na técnica de grupo focal, as informações são coletadas a partir da interação grupal, onde se promove uma ampla problematização sobre um tema ou foco específico ${ }^{(7)}$. Utilizaram-se como atividades lúdicas desenhos, jogos e dança. As conversas entre as crianças foram estimuladas por meio de questões disparadoras como: Este remédio tem gosto de....; Eu preciso tomar o remédio porque...; Quando eu não tomo o remédio o que acontece.......; sendo os encontros gravados em áudio para posterior transcrição.

Para atender a resolução 196/96 do Conselho Nacional de Saúde (CNS) a coleta das informações foi iniciada somente após a aprovação do projeto (nº 2007808 e n ${ }^{\circ}$ 001.033067.06.6) pelos Comitês de Ética em Pesquisa da Universidade Federal do Rio Grande do Sul e da Secretaria Municipal de Saúde de Porto Alegre respectivamente. Foi elaborado um termo de consentimento livre esclarecido aos pais ou responsável legal pelas crianças assegurando o direito a informações sobre a pesquisa, à participação voluntária, o anonimato em relação à identidade dos participantes, e a autorização para publicação dos dados. As crianças e seus responsáveis assinaram o termo após leitura e esclarecimento do mesmo. Para manutenção do anonimato os nomes dos participantes foram identificados como: S1, S2, S3 e S4.

A análise das informações, por meio da análise de conteúdo ${ }^{(6)}$ evidenciou três categorias: Modo de vida da criança com aids, Manuseio da medicação, Expectativas e sentimentos.

\section{ANÁLISE E INTERPRETAÇÃO DOS RESULTADOS}

\section{Conhecendo as crianças}

Participaram do estudo quatro crianças com idades entre nove e 11 anos, usuários de medicação ARV, sendo que um participante toma desde o seu nascimento, dois desde os cinco anos de idade e o outro há seis meses. As crianças em idade escolar têm capacidade de utilizar suas experiências prévias na avaliação e interpretação do presente, bem como já são capazes de compreender o que ocorre em suas vidas e realizar julgamentos sobre o que experenciam com base no que vêem (pensamento perceptual) para julgar com base no que imaginam (pensamento conceitual). Por isso, optou-se em crianças dessa faixa etária.

A constituição familiar das crianças configura-se da seguinte maneira: $\mathrm{S} 1$ reside com os pais e um irmão; S2 e S3 moram com a mãe, que é soropositiva e o padrasto e $\mathrm{S} 4$ mora apenas com a avó, pois não conheceu o pai e perdeu sua mãe em virtude da doença.

Todos frequentam a escola apresentando níveis escolares condizentes com a sua faixa etária. Este período começa com a entrada na esfera mais ampla de influência representada pelo ambiente escolar, que apresenta um impacto significativo sobre o desenvolvimento e os relacionamentos. Este período em que a criança se afilia a colegas de mesma faixa etária e aprende a cultura da infância. Com grupos de colegas, as crianças estabelecem os primeiros relacionamentos íntimos fora do grupo familiar ${ }^{(8)}$.

\section{Modo de vida da criança com AIDS}

Nesta categoria encontramos o dia-a-dia das crianças do estudo. De maneira geral percebemos nos depoimentos que o modo de vida relatado por elas é considerado "normal". As falas de S3 e S4 ilustram essa percepção:

\section{A minha vida é normal tomando remédio.(S3)}

É tudo normal. Tudo que as outras crianças fazem! Mas além de brincar eu também estudo! Então aqui eu coloquei tudo o que eu faço: eu brinco, eu corro, vejo televisão e também estudo pra ganhar boas notas.(S4)

As questões que têm de ser confrontadas nas crianças com aids, incluem aquelas em torno das 
suas características concretas, processos de pensamento e de seu desejo de ser como os seus pares, ou seja, aqueles que não têm que tomar a medicação. A omissão da condição de soropositividade, por exemplo, pode servir como estratégia, em alguns momentos, para evitar as situações de discriminação e processos de estigmatização ${ }^{(9)}$.

As crianças do estudo referiram ocultar sua condição de soropositividade perante os amigos, vizinhos, colegas de escola e alguns parentes ${ }^{(10)}$. Nas vezes em que é difícil, ou não é possível esconder a ingestão diária de medicamentos, na escola, por exemplo, utilizam estratégias para justificar essa ingestão. Esse é o caso de $\mathrm{S} 1$, o qual quando indagado se os colegas sabiam que ele tomava medicamentos diariamente, respondeu:

\section{Sabem, mas eu digo que é pro sistema ósseo. (S1)}

Essa ocultação não é incomum sendo influenciada pela própria sociedade, que por vezes considera que ter HIV/aids é algo inaceitável e que deve ser mantido em segredo. "Em nossa cultura a pessoa vivendo com HIV/aids é tida com quem tem, algo tão terrível que é obrigado a manter segredo sobre $\mathrm{si}^{(11)}$.”

Pelo que se pode depreender dos depoimentos parece existir um verdadeiro pacto de silêncio entre as crianças que vivem com HIV, como refere $\mathrm{S} 1$ :

Ninguém fala pra ninguém [que tem a doença $]\left(S_{1}\right)$

Essa situação de algo proibido, que não pode ser revelado para ninguém demonstra o temor de ser identificado com algo que é inaceitável, e por isso, ser estigmatizado, excluído do grupo e ser visto como um diferente. Desta forma, o enfrentamento da aids ultrapassa o âmbito do tratamento e esperança em relação à cura, insere-se no contexto de busca por ações de educação da população no que se refere à desmistificação dos medos e preconceitos a que as pessoas infectadas pelo HIV estão expostas ${ }^{(12)}$.

Quanto a ingerir a medicação diariamente, as crianças do estudo apresentam diferentes estratégias para não esquecer, como por exemplo, S4, que refere que utiliza programas de televisão, como marco para o horário da medicação e o celular, nos dias em que tais programas não são exibidos:
Eu coloco o celular pra despertar ou eu tomo quando começa a novela às sete horas. Daí quando vai começar a novela eu vou lá e tomo! (S4)

Essa associação do horário da medicação com uma atividade diária pode contribuir para a ingestão da medicação diariamente nos horários adequados. Nesse sentido, os profissionais de saúde podem ser importantes instrumentos para melhorar a adesão auxiliando o paciente a encontrar estratégias para o não esquecimento da medicação. Esses profissionais podem ajudá-los a selecionar uma atividade, um programa de TV ou qualquer outro meio, que faça parte do seu dia-a- dia, para associá-lo a tomada da medicação, criar grupos de apoio, qualificando a equipe, entre outras ${ }^{(13)}$.

As crianças expressaram sobre a revelação do diagnóstico como algo que acontece a partir de questionamentos decorrentes do uso das medicações. É uma situação complexa e desafiadora para os familiares e/ou cuidadores e para os profissionais de saúde:

[...] a minha mãe me falou que eu tinha que tomar um remédio, daí ela chegou com o remédio e me mandou tomar e não me disse pra que que era, só um tempinho depois eu perguntei." (SI)

No depoimento de $\mathrm{S} 1$, percebe-se que ele não tinha conhecimento das razões pelas quais deveria ingerir a medicação, ou seja, ainda não sabia de sua condição de portador do HIV.

Em um estudo, com adolescentes de São Paulo e Santos, investigando os aspectos relacionados a revelação do diagnóstico, os resultados apontaram que após um impacto negativo inicial, a revelação do diagnóstico pode favorecer o fortalecimento do vínculo do adolescente com o serviço de saúde e melhorar o diálogo com os pais e/ou cuidadores ${ }^{(14)}$. A revelação do diagnóstico é uma questão crítica e multifacetada em crianças. Nesse processo, o apoio e o envolvimento de uma equipe multidisciplinar, auxilia cuidadores em adaptar continuamente a discussão para atender às necessidades de desenvolvimento e compreensão de cada criança ao longo do tempo ${ }^{(15)}$.

Neste estudo a questão da revelação do diagnóstico não foi enfrentada de forma tão negativa ou mesmo dolorosa por parte dos sujeitos. Acredita-se que isso se deva ao fato deles serem crianças muito jovens quando lhes foi revelado, e não terem 
ainda um grau de maturidade para entenderem o significado e as prováveis implicações da doença no curso de suas vidas. Mas, são apenas inferências dos autores a essa questão, pois sabe-se que crianças portadoras de doenças crônicas ou mesmo graves apresentam uma maturidade precoce. Assim, a doença crônica impõe modificações na vida da criança e sua família, exigindo readaptações frente à nova situação e estratégias para o enfrentamento. Esse processo depende da complexidade e gravidade da doença, da fase em que eles se encontram e das estruturas disponíveis para satisfazer suas necessidades e readquirir o equilíbrio ${ }^{(16)}$.

\section{Manuseio da medicação}

Nesta categoria encontraram-se as estratégias utilizadas pelas crianças para manusear, identificar e armazenar suas medicações, as quais, foram orientadas no serviço de saúde que fazem acompanhamento. Uma estratégia relatada e, importante principalmente quando se trata de crianças, é o uso de artifícios para diferenciar a medicação que deve ser ingerida em determinados horários do dia. O sujeito $\mathrm{S} 3$ revelou contar com o auxílio de adesivos para identificar mais facilmente os medicamentos que deve ingerir no horário determinado, como ele comenta:

Eu tomo as vezes, mas as vezes eu não lembro. E o que eu tomo de noite tem o Garfield dormindo.(S3)

No serviço são fornecidos adesivos com desenhos infantis mais especificamente animais os quais as crianças colocam nos frascos de medicação para diferenciá-los e associá-los a horários estabelecidos. Por exemplo, o desenho do gatinho Garfield, nesse caso, foi colocado no frasco da medicação para ser ingerida à noite. Outro ponto importante é assegurar-se que a família e a criança tenham condições de compreender corretamente o tratamento prescrito. Não se tem como afirmar que idade seria a mais apropriada para que uma criança tivesse essas condições. Porém, a literatura aponta que entre sete e 11 anos a criança desenvolve a capacidade para operações mentais internas para resolver os problemas que surgem ${ }^{(17)}$. Ela lida melhor com conceitos, distingue a realidade da fantasia e classifica objetos ${ }^{(17)}$. Portanto acredita-se que ela tem capacidade de compreender a importância do tratamento. Sabe-se que familiares com baixo nível de instrução necessitam de informações mais claras e objetivas além de recursos que auxiliem na memorização e administração adequada dos medicamentos. Igualmente importante é informar e orientar sobre os efeitos adversos para prevenir interrupções desnecessárias do tratamento ${ }^{(3)}$.

\section{Expectativas e Sentimentos}

Nesta categoria ficou evidente o desejo de ingerir a medicação na expectativa de prolongar a vida, ou seja adiar a morte.

\section{Pra evitar a morte tão rápido. (S1)}

Se eu tomo eu não vou morrer, daí eu fico feliz! (S2)

Eu tomo porque tem que tomar. (S3)

Porque é bom pra saúde. Minha mãe faleceu porque não tomava os remédios! (S4)

A idéia de morte relacionada ao $\mathrm{HIV} /$ aids ainda persiste, mesmo com os inúmeros avanços científicos nesse $\operatorname{campo}^{(9)}$. Os participantes do estudo, mesmo sendo crianças, relacionam aids/morte e vêem na medicação a "salvação" para a vida, como percebemos, acima, na fala de S2. Essa relação ocorre, principalmente em função do fato dessas crianças já terem acompanhado o evento do "morrer com aids", em suas próprias famílias, como por exemplo, $\mathrm{S} 2$ e S3 que tiveram a experiência do falecimento do pai e $\mathrm{S} 4$ de sua mãe. A criança sabe mais a respeito da morte do que geralmente os adultos supõem. A idade cronológica, o desenvolvimento cognitivo e situações de confronto com a morte são importantes na aquisição dos conceitos de irreversibilidade (o corpo não vive após a morte), não-funcionalidade (cessação das atividades do corpo após a morte) e universalidade (tudo que é vivo morre) ${ }^{(18)}$.

Em relação a manifestação de sentimentos, o de vergonha gerado pelo preconceito as pessoas que vivem com HIV ficou bem evidente como aparece nos depoimentos abaixo:

Lá na escola se eu falo pra um, acaba espalhando. Eles começam a te deixar de lado. Lá na minha escola se souberem é preconceito! Os professores não, mais é meus amigos. (S1)

Eu não tomo, tenho bastante vergonha! Por causa do preconceito. (S3) 
Os dramas humanos e sociais são vividos no cotidiano pessoal, institucional e da sociedade, em virtude do preconceito, estigma, medo da morte, solidão e silêncio, entre outras coisas ${ }^{(19)}$. Dessa maneira, a criança e sua família vivem em seus contextos de vida situações complexas e de difícil enfrentamento necessitando dos profissionais da saúde, da educação, de outros familiares, gestores, políticos, enfim, de uma rede de suporte capaz de minimizar os dramas vividos. Não fosse o medo e a discriminação, poderia se dizer que as crianças levam sim, uma vida normal e com expectativas $^{(20)}$.

\section{CONSIDERAÇÕES FINAIS}

Trabalhar com crianças com HIV/aids no que se refere a adesão aos ARVs constitui-se ainda um desafio para os profissionais da saúde. Não se tem ainda um mecanismo que nos ajude a interpretar corretamente uma adesão nessa faixa etária, a não ser os relatos das próprias crianças e de seus cuidadores. Portanto estudos desse porte precisam ser desenvolvidos com o objetivo de auxiliar os profissionais que lidam com essa temática a conhecer e descobrir estratégias voltadas para o sucesso da adesão. Conhecer como a criança que vive com aids descreve a sua experiência com o uso de antirretrovirais possibilitou aos pesquisadores uma aproximação com a temática por meio das perspectivas da própria criança. Isso proporcionou obter um conhecimento da realidade da criança, que ultrapassa o informado pelo familiar ou profissional da saúde. Mesmo que as informações tenham sido fornecidas pelas crianças de forma simples, foi possível avaliar a profundidade das respostas o que leva a reflexões que este estudo não esgotou.

Para realização deste estudo houve algumas limitações, como a captação das crianças, pois o número inicial de sujeitos contatados foi de nove. Esse número se deve a dificuldade em agrupar as crianças e seus cuidadores em datas pré agendadas. Muitos encontros foram marcados e as crianças não compareceram por dificuldades de seus cuidadores, como a não liberação do trabalho, atividades em fins de semana e outros como por exemplo, o clima frio e chuvoso do estado em maio e junho, período do estudo interferiram no deslocamento. Além disso, o receio dos cuidadores com as doenças res- piratórias, típicas deste período, podem justificar o absenteísmo às consultas e por conseguinte às atividades propostas.

Em relação ao uso da técnica de grupo focal com crianças, essa, ainda pouco utilizada no meio acadêmico, foi uma experiência nova para os pesquisadores, que perceberam que precisam de outras vivências desse tipo para aprofundar seus conhecimentos. Acredita-se que o desenvolvimento da técnica de grupo focal em outros estudos que tenham crianças e adolescentes como sujeitos pode vir a auxiliar pesquisadores interessados na área do HIV/aids a contribuir com alternativas para o sucesso desse tratamento.

Uma das principais contribuições dessa pesquisa está em conhecer a vida da criança que tem o diagnóstico de HIV/aids, e assim possibilitar um acompanhamento de maior qualidade e mais eficaz pela equipe de saúde, o que está diretamente relacionado à melhor adesão ao tratamento.

\section{REFERÊNCIAS}

1 Ministério da Saúde (BR), Secretaria de Vigilância em Saúde. Programa Nacional de DST e Aids. Boletim Epidemiológico Aids/DST. Brasília (DF); 2011.

2 Ministério da Saúde (BR), Secretaria de vigilância em Saúde. Programa Nacional de DST e AIDS. Guia de tratamento clínico da infecção pelo HIV em crianças 2002-2003. Brasília (DF); 2003.

3 Rubini N. Adesão à terapia anti-retroviral em crianças e adolescentes. Ação Anti AIDS. 2003:49:3.

4 Leite SN, Vasconcellos MPC. Adesão à terapêutica medicamentosa: elementos para a discussão de conceitos e pressupostos adotados na literatura. Ci Saúde Col. 2003; 8(3): 775-782.

5 Polit DF, Beck CT, Hungler BT. Fundamentos da pesquisa em enfermagem: método, avaliação e utilização. Porto Alegre: Artmed; 2004.

6 Minayo M. O desafio do conhecimento: Pesquisa qualitativa em saúde. $12^{\text {a }}$ ed. Rio de Janeiro: Hucitec; 2010.

7 Backes DS, Colomé JS, Erdmann RH, Lunardi VL. Grupo focal como técnica de coleta e análise de dados em pesquisas qualitativas. O Mundo da Saúde. 2011 ;35(4):438-442. 
8 Hockenberry MJ, Winkelstein W. Wong fundamentos da enfermagem pediátrica. $7^{\mathrm{a}} \mathrm{ed}$. São Paulo: Elsevier; 2006.

9 Oleske JM. Review of recent guidelines for antiretroviral treatment of HIV-infected children. Top HIV Med. 2003;11(6):180-4.

10 Ayres PJR, França Jr I, Paiva V. Crianças e jovens vivendo com HIV/Aids: estigma e discriminação. ComCiência.2006;76.

11 Cruz EF. Infâncias, adolescências e AIDS. Educ Rev. 2007; 46: 363-384.

12 Paula CC, Padoin SMM, Vernier ETN, Motta MGC. Reflexões acerca do ser-criança e do cuidado em enfermagem no contexto da AIDS. Rev Gaúcha Enferm. 2003;24(2):189-95.

13 Kourrouski MFC, Lima RAG. Adesão ao tratamento: vivências de adolescentes com HIV/AIDS. Rev Latino-Am Enferm. 2009; 17(6):947-952.

14 Marques HHS, Silva NG, Gutierrez PL, Lacerda R, Ayres JRCM, DellaNegra M, et al. A revelação do diagnóstico na perspectiva dos adolescentes vivendo com HIV/AIDS e seus pais e cuidadores. Cad Saúde Pública. 2006; 22(3): 619-629.

15 Domek GJ. Debunking Common Barriers to Pediatric HIV Disclosure. J Trop Pediatr. 2010;56(6):440-2.

16 Vieira MA, Lima RAG. Crianças e adolescentes com doença crônica: convivendo com mudanças. Rev Latino-Am. Enferm. 2002; 10(4):552-560.

17 Papalia DE, Olds SW, Feldman RD. Desenvolvimento Humano. $8^{\mathrm{a}}$ ed. Porto Alegre: Artmed; 2006.

18 Torres WW. O conceito de morte em crianças portadoras de doenças crônicas. Psic: Teor Pesq. 2002; 18(2): 221-229.

19 Gomes AMT, Cabral IE. Ocultamento e silenciamento familiares no cuidado à criança em terapia antirretroviral. Rev Bras Enferm. 2010;63(5):719726.

20 Pedro ENR; Stobäus CD. Vivências e (con)vivências de crianças portadoras do HIV/AIDS e seus familiares; implicações educacionais. Rev Paulista Enferm. 2003;22(1): 62-71.

\section{Endereço do autor / Dirección del autor / Author's address}

Joel Kuyava

Avenida da Azenha, 680, ap.1306, Azenha

90160-005, Porto Alegre, RS

E-mail:jkuyava@yahoo.com.br
Recebido em: 18.10.2011

Aprovado em: 31.08 .2012 